TITLE:

\title{
PROJECTIVE-PLANAR GRAPHS WITH EVEN DUALS
}

$\operatorname{AUTHOR}(\mathrm{S}):$

NEGAMI, Seiya

CITATION:

NEGAMI, Seiya. PROJECTIVE-PLANAR GRAPHS WITH EVEN DUALS. 数理 解析研究所講究録 1991, 768: 131-138

ISSUE DATE:

1991-11

URL:

http://hdl.handle.net/2433/82321

RIGHT: 


\title{
PROJECTIVE-PLANAR GRAPHS WITH EVEN DUALS
}

\author{
Seiya NEGAMI \\ Department of Mathematics, Faculty of Education \\ Yokohama National University \\ 156 Tokiwadai, Hodogaya-Ku, Yokohama 240, Japan
}

\section{Introduction}

As is well-known, a graph is bipartite if and only if it contains no odd cycle. So if we embed a bipartite graph $G$ on a closed surface $F^{2}$, then the boundary walk of each face has even length. We call such a face simply an even face. In this case, the dual of $G$ embedded in $F^{2}$ is even, that is, each vertex of the dual has even degree.

Also it is well-known that a planar graph has an embedding with only even faces in the sphere or the plane if and only if it is bipartite. For any eulerian map on the sphere or the plane is 2-colorable. However, the necessity does not hold for other surfaces in general since an odd cycle might be hidden as one that is not null-homotopic in the surface. In this paper, we discuss those graphs which have even duals in closed surfaces in Section 2 and characterize such projective-planar graphs in Section 3.

To discribe our main theorem, we define the canonical bipartite covering, denoted by $B(G)$, for any graph $G$ as follows: When $G$ is not bipartite, we prepare two vertices $v_{1}$ and $v_{2}$ for each vertex $v \in V(G)$ and add two edges $v_{1} u_{2}$ and $v_{2} u_{1}$ if there is an edge $v u \in E(G)$. The resulting graph is $B(G)$ in this case and it is a bipartite graph with partite sets $\left\{v_{1}: v \in V(G)\right\}$ and $\left\{v_{2}: v \in V(G)\right\}$. When $G$ is bipartite, we set $B(G)=G$.

THEOREM 1. Let $G$ be a connected projective-planar graph but nonplanar. Then $G$ has a projective-planar embedding with only even faces if and only if either $G$ is bipartite or $B(G)$ is planar.

For example, consider the Möbius ladder $O_{n}$ which is defined as a cycle $v_{1} v_{2} \cdots v_{2 n}$ of length $2 n$ with $n$ diagonals $v_{i} v_{i+n}$. It is easy to see that $O_{n}$ is bipartite if and only if $n$ is odd. When $n$ is even, that is, when $O_{n}$ is not bipartite, $B\left(O_{n}\right)$ is isomorphic to $C_{2 n} \times K_{2}$ and is planar. Thus, $O_{n}$ can be embeddable in the projective plane so that it has only even faces by Theorem 1 .

Actually $O_{n}$ has two inequivalent projective-planar embeddings given in Figure 1. (Each pair of antipodal points on the boundary circle should be identified to get the projective plane.) The left one has an even dual while the right one does not if $n$ is even. 

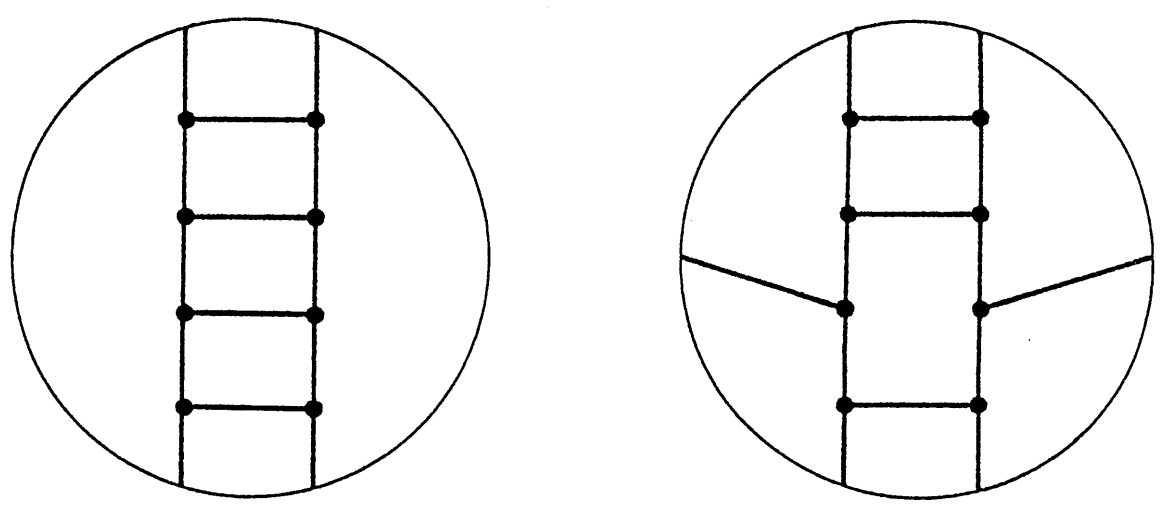

Figure 1: Möbius ladders

As this example shows, a graph might have a projecrive-planar embedding without an even dual even if $B(G)$ is planar. We can however show the uniqueness of even duals, as follows:

THEOREM 2. A 3-connected projective-planar graph $G$, not planar, has a unique even dual in the projective plane if $B(G)$ is planar.

This can be said to correspond to the well-known fact that every 3-connected planar graph has a unique dual in the sphere, proved by Whitney [6]. As is pointed out in [1], his result is equivalent to that every 3 -connected planar graph is uniquely and faithfully embeddable in the sphere. The uniqueness and the faithfulness of planar embeddings will play an important role to prove our theorems via the notion of covering spaces as well as in proofs of the results given in [2] and [4].

\section{General Observations}

A connected graph $\tilde{G}$ is called an ( $n$-fold) covering of a connected graph $G$ if there is an (n-to-one) surjection $p: V(\tilde{G}) \rightarrow V(G)$, called its projection, which induces bijections between neighborhoods of corresponding vertices. Such a projection $p: V(\tilde{G}) \rightarrow V(G)$ extends naturally to a map between edge sets by $p(u v)=p(u) p(v)$. We denote simply $p: \tilde{G} \rightarrow G$ to express these two projections and often regard it as a local homeomorphism from $\tilde{G}$ to $G$ in the topological sense. Although the covering spaces of graphs can be defined combinatorially as above, we shall attempt here to discuss covering spaces of graphs in terms of algebraic topology. (See standard text books of algebraic topology, for example [5], if you want to know the general arguments on covering spaces.)

By the classification of covering spaces, there is a one-to-one correspondence between the equivalence classes of coverings of $G$ and the conjugacy classes of subgroups of its fundamental group $\pi_{1}(G)$. If a covering $p: \tilde{G} \rightarrow G$ is associated with a subgroup $H$, then the fold number of $p$ is equal to the number of cosets of $H$ in $\pi_{1}(G)$. In particular, if $H$ is normal in $\pi_{1}(G)$, then the quotient group $\pi_{1}(G) / N$ acts on $\tilde{G}$ so that any two vertices in $\tilde{G}$ which project to the same vertex belong to the same orbit. Such a covering is called a 
regular covering and the group $\pi_{1}(G) / N$ acting on $\tilde{G}$ is called the covering transformation group of $p: \tilde{G} \rightarrow G$.

The canonical bipartite covering $B(G)$ can be defined topologically as the one associated with the subgroup $N$ in $\pi_{1}(G)$ which consists of all the closed walks in $G$ of even length. Such a subgroup $N$ is normal and has index 2 in $\pi_{1}(G)$ if $G$ is not bipartite while $N=\pi_{1}(G)$ if $G$ is bipartite. Thus, $B(G)$ is a 1- or 2 -fold covering of $G$. In general, a closed walk $W$ in $G$ can be lifted to a closed walk in $\tilde{G}$ if and only if the homotopy class of $W$ belongs to the subgroup $H$ which $\tilde{G}$ is associated with; otherwise, we would obtain a walk in $\tilde{G}$ with distinct ends, tracing edges of $W$ via $p$. This fact implies that $B(G)$ contains no odd cycle and hence $B(G)$ is bipartite.

The following lemma shows the most important property of $B(G)$ and has been proved in [3] by two methods, combinatorial or topological.

LEMMA 3. Every bipartite covering $p: \tilde{G} \rightarrow G$ factors through the canonical bipartite covering $B(G)$ of $G$. That is, there is a covering projection $q: \tilde{G} \rightarrow B(G)$ with $p=b q$, where $b: B(G) \rightarrow G$ is the covering projection from $B(G)$ to $G$.

Notice that $G$ has a unique 2 -fold bipartite covering, which is nothing but $B(G)$, when $G$ is not bipartite; if $p: \tilde{G} \rightarrow G$ is a 2 -fold bipartite covering, then $q: \tilde{G} \rightarrow B(G)$ has to be an isomorphism between them.

The following theorem generalizes Theorem 1 but shows only the necessity:

THEOREM 4. Let $G$ be a connected graph 2-cell embedded in a closed surface $F^{2}$. If the dual of $G$ in $F^{2}$ is even, then either $G$ is bipartite or $B(G)$ is embeddable in a 2-fold covering space of $F^{2}$.

Proof. Let $f: G \rightarrow F^{2}$ be the embedding of $G$ into $F^{2}$. Then we have the following short exact sequence induced by the surjective homomorphism $f_{*}: \pi_{1}(G) \rightarrow \pi_{1}\left(F^{2}\right)$ :

$$
1 \longrightarrow \operatorname{ker} f_{*} \longrightarrow \pi_{1}(G) \longrightarrow \pi_{1}\left(F^{2}\right) \longrightarrow 1
$$

The kernel ker $f$ can be regared to be generated by the boundary walks of faces of $f(G)$. (More precisely, they need headers and tails which connect them to the base point of $\pi_{1}(G)$.)

Let $N$ be the normal subgroup of $\pi_{1}(G)$ consising of only closed walks of even length. Then the dual of this embedding is even if and only if

$$
\operatorname{ker} f_{*} \subset N \text {. }
$$

Suppose that $G$ is not bipartite. Then the normal subgroup $N$ has index 2 in $\pi_{1}(G)$. If $f_{*}(N)=\pi_{1}\left(F^{2}\right)$, then any odd cycle $f(C)$ of $f(G)$ is homotopic to a closed walk $f(W)$ of even length on $F^{2}$ and hence $f(C) f(W)^{-1}$ is null-homotopic walk of odd length. This implies that $C W^{-1}$ belongs to ker $f$ and hence it can be decomposed into a product of face boundary walks, at least one of which should have odd length.

Therefore, if the dual of $f(G)$ is even, then $f_{*}(N)$ does not coincide with $\pi_{1}\left(F^{2}\right)$ and has index 2 in $\pi_{1}\left(F^{2}\right)$. So we can take the 2 -fold covering $p: \tilde{F}^{2} \rightarrow F^{2}$ associated with $f_{*}(N)$. Then the lift $\tilde{G}=p^{-1}(f(G))$ of $f(G)$ covers $f(G)$ and also $G$ doubly. By the 
definition of $N$, only closed walks of even length can be lifted to $\tilde{G}$. This implies that the 2 -fold covering $\tilde{G}$ is bipartite and hence it has to be isomorphic to $B(G)$ by Lemma 3. Thus, $B(G)$ is embeddable in $\tilde{F}^{2}$.

If $\tilde{F}^{2}$ covers $F^{2} n$-fold, then $\chi\left(\tilde{F}^{2}\right)=n \chi\left(F^{2}\right)$, where $\chi(*)$ denotes the Euler number and any nonorientable surface cannot cover an orientable one. Thus, any two 2-fold covering spaces of an orientable closed surface are hoemomorphic to each other. For example, any 2 -fold covering of a torus is also a torus. So the above theorem can read for a toroidal graph $G$ that if $G$ has an even dual in the torus, then either $G$ is bipartite or $B(G)$ is toroidal. On the other hand, a nonorientable closed surface has exactly two 2 -fold covering spaces, orientable or nonorientable, unless it is the projective plane. For example, the torus and the Klein bottle cover the Klein bottle doubly while the only sphere covers the projective plane. These facts imply that the condition of $B(G)$ in Theorem 4 is not so ambiguous.

Examples: The canonical bipartite covering $B\left(K_{n}\right)$ of the complete graph $K_{n}$ is isomorphic to the complete bipartite graph $K_{n, n}$ with a perfect matching deleted. For example, $B\left(K_{4}\right)$ is planar and $K_{4}$ have a projective-planar embedding with three rectangular faces, and hence with an even dual but not simple. The complete graph $K_{5}$ is projective-planar but $B\left(K_{5}\right)$ is nonplanar since it contains $K_{3,3}$ with one edge subdivided by two vertices. Thus, $K_{5}$ does not have any even dual in the projective plane while it has an even dual in the torus, isomorphic to $K_{5}$ itself, and hence $B\left(K_{5}\right)$ is toroidal. For $n \geq 6, B\left(K_{n}\right)$ is not toroidal since it contains two disjoint $K_{3,3}$ 's. Thus, neither $K_{6}$ nor $K_{7}$ have even duals in the torus although they are embeddable there.

\section{Projective-Planar Case}

There are two important notions to discuss on projective-planar graphs, as the arguments in [2] and [4] suggest; the first is to connect projective-planar embeddings and planar coverings and the second is the uniqueness and faithfulness of 3-connected planar graphs, defined first in [1], as follows. Two embeddings $f, g: G \rightarrow F^{2}$ are said to be equivalent here if there exist an automorphism $\sigma: G \rightarrow G$ and a homeomorphism $h: F^{2} \rightarrow F^{2}$ with $h f=g \sigma$. A graph $G$ is said to be uniquely embeddable in $F^{2}$ if $G$ has only one equivalence class of embeddings into $F^{2}$. On the other hand, A faithful embedding $f: G \rightarrow F^{2}$ is such a one that for any automorphism $\sigma: G \rightarrow G$, there is a homeomorphism $h: F^{2} \rightarrow F^{2}$ with $h f=f \sigma$, and $G$ is said to be faithfully embeddable in $F^{2}$ if $G$ has such an embedding.

As is well-known, evey 3-connected planar graph has a unique dual in the sphere and it can be said in our terminology that it is uniquely and faithfully embeddable in the sphere. Suppose that a given planar covering $\tilde{G}$ is regular and 3-connected. Then we can embed $\tilde{G}$ in the sphere $S^{2}$ so that its covering transformation group $\Gamma$ extends to a group of auto-homeomorphisms on $S^{2}$ since it is faithfully embeddable, and the orbit space $S^{2} / \Gamma$ should be the projective plane, where $G$ is embedded.

By the same way, we can get an even dual of $G$ in the projective plane if $B(G)$ is planar and 3-comnected. The assumption of $B(G)$ being 3-connected is however too strong to be 
valid in general. We need only that its covering transformations extend, but not all the automorphisms of $B(G)$.

The following three lemmas will be used to prove the extendability of covering transformations.

LEMMA 5. Let $G$ be a 2-connected graph and $\tilde{G}$ a 2-fold planar covering of $G$. Then $\tilde{G}$ can be embedded in the sphere $S^{2}$ so that its covering transformation of order 2 extends to an involution on $S^{2}$.

Proof. We use induction on the number of vertices of $G$. Let $p: \tilde{G} \rightarrow G$ be the covering projection and $\tau: \tilde{G} \rightarrow \tilde{G}$ the covering transformation. It is easy to see that $\tau$ extends to the antipodal map or the rotation around some axis through $\pi$ when $G$ has a few vertices.

Suppose that $G$ has more vertices. When $\tilde{G}$ is 3 -connected, $\tilde{G}$ is uniquely and faithfully embeddable in the sphere and hence $\tau$ extends automatically for any spherical embedding of $\tilde{G}$. On the other hand, when $\tilde{G}$ is not 3 -connected, we can reduce it to the case that $G$ has fewer vertices, as follows.

Let $U \subset V(\tilde{G})$ be a 1- or 2-cut which $\tilde{G}$ has to have. First suppose that $p(U)$ consists of only one vertex of $G$. Since $G$ is 2-connected, any two vertices in $V(G)-p(U)$ can be joined by a path which does not meet $p(U)$. This implies that $\tilde{G}-U$ consists of precisely two components and hence that $\tilde{G}$ decomposes to two subgrahps $H$ and $\tau(H)$ with $H \cap \tau(H)=U=\tau(U)$. If $U$ were a 1-cut, then the cut vertex in $U$ would be a fixed point of $\tau$, contrary to $\tau$ being a free involution. Thus, $U$ consists of two vertices which $\tau$ exchanges. In this case, $\tau$ extends to an involution on $S^{2}$ equivalent to either the antipodal map or the rotation around axis through $\pi$, depending on the embeddings of $H$ and $\tau(H)$.

Now suppose that $p(U)$ consists of two vertices of $G$. Since $G$ has no cut vertex by the above arguments, $U$ has to contains two vetices $u, v$ and $\tau(U) \cap U=\emptyset$. Then $G$ decomposes into three subgraphs $K, H$ and $\tau(H)$ such that $\tau(K)=K, K \cap H=U$ and $H \cap \tau(H)=\emptyset$ if we choose $U$ so that one of components of $\tilde{G}-U$ is minimal with respect to inclusion among all the 2-cuts of $\tilde{G}$.

Add new edges $u v$ and $\tau(u) \tau(v)$ to $K$ if they do not exist in $K$. Then $\tau$ induces a free involution $\tau_{K}$ over the resulting graph $K^{\prime}(=K+\{u v, \tau(u) \tau(v)\})$. Now $G$ decomposes into two subgraphs $p\left(K^{\prime}\right)$ and $p(H)$ which meet in $p(U)$ and $G^{\prime}=p\left(K^{\prime}\right)+p(u) p(v)$ is 2-connected. Since $K^{\prime \prime}$ covers $G^{\prime}$ doubly, $K^{\prime \prime}$ can be embedded in $S^{2}$ so that $\tau_{K}$ extends to an involution $h: S^{2} \rightarrow S^{2}$. If we embed $H$ along the arc $u v$ and $\tau(H)$ along $\tau(u) \tau(v)$, then $h$ will be an extension of $\tau$.

In either case, we have got an spherical embedding of $\tilde{G}$ with $\tau$ extendable and hence our induction completes.

LEMMA 6. Let $G$ be a connected nonplanar graph and let $\tilde{G}$ be a 2-fold planar covering of $G$ embedded in the sphere. Then no. two vertices of $\tilde{G}$ which project to the same vertex are incident to a common face.

Proof. Let $p: \tilde{G} \rightarrow G$ be the covering projection and $\tau: \tilde{G} \rightarrow \tilde{G}$ the covering transformation of order 2. Since $G$ is nonplanar, $G$ contains a subdivision $K$ of either $K_{5}$ or $K_{3,3}$ and $\tilde{K}^{\prime}=p^{-1}\left(K^{\prime}\right)$ is a 2 -fold planar covering of $K$ with transformation $\left.\tau\right|_{\tilde{K}}$. 
By Lemma 4 in [2], $\tilde{K}^{\prime}$ is a subdibision of a 3-connected planar graph. Since every 3-connected planar graph is faithfully embeddable in the sphere $S^{2},\left.\tau\right|_{\hat{K}}$ extends to an involution $h: S^{2} \rightarrow S^{2}$, which is equivalent to the antipodal map. It is not however assured whether the whole of $\tau$ extends or not.

Suppose that for some vertex $v \in V(\tilde{G})$, both $v$ and $\tau(v)$ are incident to a common face. Then thery are contained in one face $A$ of $\tilde{K}$. This implies that either $\tau(A)=A$ or $\tau(A) \cap A=\{v, \tau(v)\}$. In the former case, the face $A$ would contain a fixed point of $h$, contrary to $h$ being a free involution. In the latter case, $\tilde{K}$ splits at $\{v, \tau(v)\}$, which contradicts that $K$ is a subdivision of a 3-connected graph. Therefore, $v$ and $\tau(v)$ lie on the boundaries of distinct faces.

LEMMA 7. Let $G$ be a connected graph with blocks $B_{0}, B_{1}, \cdots, B_{n}$ and let $p: \tilde{G} \rightarrow G$ be a 2-fold planar covering of $G$. If $G$ is projective-planar but nonplanar, then precisely one of blocks, say $B_{0}$ is nonplanar and the others can be lifted to $\tilde{G}$ isomorphically.

Proof. It is clear that at least one of $B_{i}$ 's, say $B_{0}$, is nonplanar. If we embed $G=$ $B_{0} \cup B_{1} \cup \cdots \cup B_{n}$ in the projective plane, then the other blocks $B_{i}(i \geq 1)$ is embedded within faces of $B_{0}$, which are homeomorphic to 2-cells. Thus, they are planar.

Suppose that $B_{1}$ could not be lifted isomorphically. Then $p^{-1}\left(B_{1}\right)$ is a 2 -fold covering of $B_{1}$, which is connceted. Let $P$ be a shortest path connecting $B_{0}$ to $B_{1}$ with ends $u \in V\left(B_{0}\right)$ and $v \in V\left(B_{1}\right)$ and let $P^{\prime}$ and $P^{\prime \prime}$ be two distinct lifts of $P$ in $\tilde{G}$. Then the connected subgraph $P^{\prime} \cup p^{-1}\left(B_{1}\right) \cup P^{\prime \prime}$ has to be contained in a face of the 2-fold planar covering $p^{-1}\left(B_{0}\right)$ and hence the two lifts of $u$ are incident to the face together, contrary to Lemma 6.

The following theorem is just what we need to prove Theorem 1:

THEOREM 8. Let $G$ be a connected graph which is projective-planar but nonplanar and let $p: \tilde{G} \rightarrow G$ be a 2-fold planar covering of $G$ with transformation $\tau: \tilde{G} \rightarrow \tilde{G}$ of order 2. Then $\tilde{G}$ can be embedded in the sphere $S^{2}$ so that $\tau$ extends to an involution on $S^{2}$.

Proof. After unifying some blocks, we can assume that $G$ decomposes into a 2connected nomplanar subgraph $B_{0}$ and several mutually disjoint connected planar subgraphs $B_{1}, \cdots, B_{n}$ each of which meets $B_{0}$ in a cut vertex $v_{i}$. By Lemma $7, \tilde{G}$ can be obtained as the union $\tilde{B}_{0} \cup B_{1}^{\prime} \cup B_{1}^{\prime \prime} \cup \cdots \cup B_{i}^{\prime} \cup B_{i}^{\prime \prime}$, where $\tilde{B}_{0}$ is a 2 -fold planar covering of $B_{0}$ and $B_{i}^{\prime}$ and $B_{i}^{\prime \prime}$ are disjoint copies of $B_{i}(i \geq 1)$.

By Lemma 5, $\hat{B}_{0}$ can be embedded in $S^{2}$ so that $\left.\tau\right|_{B_{0}}$ extends to an involution $h$ : $S^{2} \rightarrow S^{2}$ since $B_{0}$ is 2-connected. By Lemma 6 , the two lifts $v_{i}^{\prime}$ and $v_{i}^{\prime \prime}$ of $v_{i}$ are incident to distinct faces $A$ and $h(A)$. First embed $B_{i}^{\prime}$ in $A$ and next embed $B_{i}^{\prime \prime}$ onto $h\left(B_{i}^{\prime}\right)$ for each $i$, so that $\left.h\right|_{\tilde{G}}$ realizes $\tau$.

Note that the assumption of $G$ being nonplanar cannot be omitted from Theorem 8 . For example, let $H_{n}$ be the graph obtained as a comnected planar graph $H$ with $n$ disjoint triangles added to a face boundary at one vertex each. Then $H_{n}$ has a 2-fold planar covering $\tilde{H}_{n}$ which can be obtained as two copies of $H$ joined by $n$ hexagons. Its covering transformation $\tau: \tilde{H}_{n} \rightarrow \tilde{H}_{n}$ fixes each hexagon setwise. To realize this action on the $n$ 
hexagons, we have to place them as concentric circles on the sphere, so that the rotation around the center through $\pi$ acts on them. If $n \geq 3$, then we cannot add two $H$ 's to get a planar embedding of $\tilde{H}$. Since $H_{n}$ is not 2-connected. this example also shows that $G$ has to be 2-connected in Lemma 5 .

Suppose that $H_{n}$ had a projective-planar embedding with an even dual. Then the $n$ triangles would be essential cycles, but any two essentail cycles have to meet each other in the projective plane. Thus, $H_{n}$ with $n \geq 2$ has no even dual in the projective plane even if $B\left(H_{n}\right)=\tilde{H}_{n}$ is planar when $H$ is bipartite. That is, such an $H_{n}$ is a counterexample to Theorem 1 with the nonplanarity of $G$ omitted.

Proof of Theorem 1. The necessity follows from Theorem 4 since the only 2 -fold corering space of the projective plane is the sphere.

To show the sufficiency, we suppose that $G$ is not bipartite and that $B(G)$ is planar. Then $B(G)$ is a 2-fold covering of $G$ and has the covering translation $\tau: B(G)-B(G)$ of order 2. By Theorem $S, B(G)$ can be embedded in the sphere $S^{2}$ so that $\tau$ extends to a free involution $h: S^{2} \rightarrow S^{2}$, equivalent to the antipotal map of $S^{2}$; otherwise, $G$ would be planar.

The orbit space $S^{2} / h$ is homeomorphic to the projective plane $P^{2}$ and contains a projective planar embedding of $G$. If $h$ fixed a face setwise, there would be a fixed point within the face by Brawer's fixed point theorem, contrary to $h$ being free. Thus, $h$ carries each face of $B(G)$ onto another different one and the covering projection $p: S^{2} \rightarrow P^{2}(=$ $\left.S^{2} / h\right)$ sends it onto a face of $G$ homeomorphically. Since $B(G)$ is bipartite, $B(G)$ has only even faces and hence so does $G$.

Proof of Theorem 2. Let $f: G \rightarrow P^{2}$ be any projective-planar embedding with an even daul and let $p: S^{2} \rightarrow P^{2}$ be the 2 -fold covering of the projective plane $P^{2}$. Then $p^{-1}(f(G))$ is a 2-fold covering of $G$ and also is embedded in the sphere $S^{2}$ with an even daul. This implies that $p^{-1}(f(G))$ is bipartite and has to be isomorphic to $B(G)$ by Lemma 3 .

Negami has shown in [2] that there is a bijection between the equivalence classes of projective-planar embeddings and the isomorphism classes of 2-fold planar corerings of $G$. Thus, $f$ is equivalent to the one derived from a planar embedding of $B(G)$ in the proof of Theorem 1, which implies that any two even duals are isomorphic to cach other via. $B(G)$.

\section{References}

[1] S. Negami, Uniqueness and faithfulness of embedding of toroidal graphs. Discrete Math. 44 (1983), 161-180.

[2] S. Negami, Enumeration of projective-planar embeddings of a graph, Discrete Math. 62 (1986), 299-306.

[3] S. Negami, The virtual k-factorability of graphs, J. Graph Theory 11 (1957), 359365. 
[4] S. Negami, The spherical genus and virtually planar graphs, Discrete Math. 70 (1988), 159-168.

[5] E. Spanier, "Algebraic topology", McGraw-Hill Book Company, 1966.

[6] H. Whitney, Congruent graphs and the connectivity of graphs, Amer. J. Math. 54 (1932), 150-168. 